\title{
Resistant virulent Candida species colonizing preterm neonates and in vitro promising prospect of chlorhexidine gluconate
}

\author{
Iman E. Wali ${ }^{1 \star}$, Rasha H. Bassyouni ${ }^{2}$, Eman Ahmed El-Seidi ${ }^{1}$, Amira Edris ${ }^{3}$, Abdel-Rahman \\ Ahmed Abdel-Razek ${ }^{3}$, Amina Abdel-Salam ${ }^{3}$ and Reem Fouad ${ }^{4}$ \\ ${ }^{1}$ Medical Microbiology and Immunology Department, Faculty of Medicine, Cairo University, Egypt. \\ ${ }^{2}$ Medical Microbiology and Immunology Department, Faculty of Medicine, Fayoum University, Egypt. \\ ${ }^{3}$ Department of Pediatrics, New Children's Hospital, Cairo University, Egypt. \\ ${ }^{4}$ The Ministry of Health, Cairo, Egypt.
}

Accepted 12 June, 2013

\begin{abstract}
The present study aimed to investigate the potential virulence factors and antifungal resistance of $\mathbf{3 1}$ Candida albicans and 21 non-albicans Candida isolates colonizing preterm neonates. The study also compared the susceptibility results with the in vitro activity of chlorhexidine in the eradication of Candida colonization. Candida albicans produced significantly more phospholipase and coagulase than non-albicans Candida, whereas proteinase production was higher in non-albicans Candida. Biofilm production was demonstrated in Candida albicans and non-albicans Candida $(P=0.214)$. None of the planktonic growth of Candida isolates were resistant to either fluconazole or amphotericin B, whereas $40 \%$ and $84 \%$ Candida isolates grown as biofilm became resistant to fluconazole and amphotericin B, respectively. Both coagulase and phospholipase production strongly correlated with the resistance of sessile Candida isolates to amphotericin $B(P<0.001)$. Whereas both proteinase and phospholipase correlated with the resistance of in vitro Candida biofilms to fluconazole $(P<0.05$ and $P$ $=0.001$; respectively). Chlorhexidine was comparable to fluconazole towards planktonic and sessile grown Candida isolates. In conclusion, the study demonstrated an association between certain virulence factors and the development of biofilm drug resistance and highlighted the value of chlorhexidine as a promising prospect in the eradication of Candida colonization.
\end{abstract}

Key words: Antifungal susceptibility, biofilm resistance, Candida colonization, chlorhexidine, preterm neonates, virulence factors.

\section{INTRODUCTION}

Systemic fungal infections, mainly by Candida species, are the third most frequent cause of late-onset sepsis among very low birth weight preterm neonates in neonatal intensive care units (NICUs) (Manzoni et al., 2011). Skin and gastrointestinal tract colonization by Candida species in neonates is necessary for the pathogenesis of invasive Candida infections (ICls) (Mendiratta et al.,
2006). Candida colonization and subsequent infection depend on many different virulence phenotypes such as adhesion to host cells, hyphae formation, hydrolytic enzymes production (such as phospholipase as well as secretory aspartyl proteinase) and biofilm production (Mohan das and Ballal, 2008). Moreover, Candida biofilms areknowntoexhibitelevatedantifungal resistancecompared 
to their planktonic counterparts (Seneviratne et al., 2008). Amphotericin B (ampB) deoxycholate is a primary antimicrobial medication for proven or suspected neonatal invasive fungal infection. It is generally better tolerated in neonates than in adults; since ampB has a longer half-life, lower serum level and faster elimination in neonates unless there is a rise in the serum creatinine and blood urea nitrogen (Turkova et al., 2011).

Fluconazole (FCZ), on the other hand, should be reserved for prophylaxis, though it is considered as an alternative to amphotericin B in the treatment of neonatal candidiasis. However, the use of fluconazole prophylaxis in NICUs has raised concerns about the development of resistance (Leibovitz, 2012).

Chlorhexidine gluconate (CHX) is a topical antiseptic used in a myriad of clinical settings (Soma et al., 2012). Prophylactic use of chlorhexidine in combination with daily oral hygiene care has been associated with reduction of potentially pathogenic microorganisms in the oral mucosa of children with acute lymphoblastic leukemia (Soares et al., 2011). Moreover, daily chlorhexidine baths applied to pediatric patients with central lines was associated with lower counts of cultivable cutaneous bacteria (Soma et al., 2012). Recently, its fungicidal activity towards certain Candida species has been shown, with suggestions of gargling with chlorhexidine mouth rinses for rapid reduction of Candida population in patients with fungal infection (Fathilah et al., 2012).

The aim of the present study was to investigate the potential virulence factors and antifungal resistance of Candida species colonizing preterm neonates. The study also sought to compare the antifungal susceptibility results with the in vitro activity of chlorhexidine as an alternative prospect in the eradication of Candida colonization.

\section{MATERIALS AND METHODS}

\section{Candida strains}

Fifty-two Candida isolates colonizing 21 out of 50 premature neonates admitted to our NICU at the Children's Hospital, Cairo University, during the study period from August till December 2010 were included in the study. The isolates were obtained by surveillance cultures of swabs taken from the oral cavity, umbilicus, groin and rectum on admission and after 7 days. The study protocol was approved by the ethical committee of our Hospital and was conducted in accordance with the University bylaws for human research.

Specimens were inoculated onto Sabouraud Dextrose Agar medium (SDA) (Oxoid Ltd., Hampshire, United Kingdom) and incubated at $37^{\circ} \mathrm{C}$ for $24 \mathrm{~h}$, then colonies were identified as Candida by Gram stained smears (Howell and Hazen, 2011).

Further identification to the species level was done by the API Candida system (Bio-Merieux,Marcy-l'Etoile, France).

Different isolates belonging to the same species within the same patient were typed by the resistogram typing method according to the study of Nakamura et al. (1998). Four concentrations were used for each of the following chemicals: sodium selenite, boric acid, cetrimide, sodium periodate, malachite green and copper sulphate; which were allotted the letters $A, B, C, D, E$ and F; respectively. Isolates were considered to belong to different strains if they demonstrated a difference in resistance to one or more chemicals. An isolate was deemed resistant to a chemical if growth was seen with three of the four concentrations used for each chemical. Accordingly, each isolate's resistogram was made of the letters representing the corresponding chemicals to which that isolate was resistant.

\section{Detection of virulence factors \\ Phospholipase and proteinase activity detection}

Secretion of phospholipase and proteinase enzymes was detected as previously described by Mohan das and Ballal (2008). Candida albicans ATCC 10231 served as a positive control. The tested isolates were classified as negative, 1+, 2+, 3+ and 4+. Assays were performed in replicates of three.

\section{Detection of coagulase activity}

Approximately $100 \mu \mathrm{l}$ of an overnight culture on Sabouraud dextrose broth (SDB) (Oxoid Ltd., Hampshire, United Kingdom) was inoculated into tubes containing $500 \mu \mathrm{l}$ rabbit plasma collected and stored as previously described (Yigit et al., 2008). The tubes were incubated at $37^{\circ} \mathrm{C}$ and observed for clot formation after $24 \mathrm{~h}$. Staphylococcus aureus ATCC 25923 and tubes containing rabbit plasma only were used as positive and negative controls respectively.

\section{Testing for pseudohyphae formation}

Equal volumes of RPMI 1640 (Sigma, -Aldrich, Dorset, UK) and fetal bovine serum (GIBCO, Invitrogen, Burlington, Ontario) were inoculated with an overnight growth culture of the tested Candida isolates. After $2 \mathrm{~h}$ of incubation at $37^{\circ} \mathrm{C}$, microscopic counting was done to determine the percentage of cells growing in pseudohyphae form against blastospores (Negri et al., 2010). Each isolate was tested in triplicate.

\section{Testing for biofilm production}

Biofilm formation was assessed spectrophotometrically at $405 \mathrm{~nm}$ as previously described by Shin et al. (2002) using 96-well tissue culture microtitre plates (Nunclon; Nalge Nunc International, Roskilde, Denmark). The assay was performed in duplicates and the isolates were scored as negative, $1+, 2+, 3+$ or $4+$.

\section{Antifungal susceptibility testing}

The minimum inhibitory concentrations (MICs) of FCZ (Diflucan $2 \mathrm{mg} / \mathrm{ml}$ IV vial, Pfizer) and ampB (Fungizone $50 \mathrm{mg} / \mathrm{ml} \mathrm{IV} \mathrm{vial,}$ Bristol-Myers Squibb) were determined against planktonic cells by the broth microdilution method in accordance to the clinical laboratory standards institute (CLSI) document M27-A3 (CLSI, 2008). The reference strain Candida parapsilosis ATCC 22019 was used as a quality control strain. The procedure was also adapted for testing serial dilutions of aqueous solution of $\mathrm{CHX}$ (Antiseptol solution $0.1 \mathrm{gm} / 100 \mathrm{ml}$; Kahira Pharm and Chem. Ind. Co., Cairo, Egypt). The tested final concentration ranged from $0.03-16 \mu \mathrm{g} / \mathrm{ml}$ for ampB and $0.12-64 \mu \mathrm{g} / \mathrm{ml}$ for $\mathrm{FCZ}$ as well as $\mathrm{CHX}$. MIC breakpoint interpretation for FCZ was done according to the CLSI guidelines (CLSI, 2008). Interpretive breakpoints have not yet been established for ampB and $\mathrm{CHX}$, but breakpoints for ampB proposed by Nguyen et al. (1998) were used to classify isolates.

Moreover, the antifungal susceptibility of the biofilm grown Candida isolates to the previously mentioned agents with the same prepared dilutions was done by the microplate alamar blue assay (Repp et al., 2007). The susceptibility results were expressed as ranges of minimum and maximum MIC values, MIC for $50 \%$ of the organisms (MIC50) and MIC for $90 \%$ of the organisms (MIC90). 
Table 1. Distribution of the isolated Candida species among different sites.

\begin{tabular}{lccccc}
\hline Candida spp. & Oral & Umbilicus & Groin & Rectum & Total (\%) \\
\hline C. albicans & $6(1)^{*}$ & 3 & $12(4)^{*}$ & $10(3)^{*}$ & $31(59.6 \%)$ \\
C. tropicalis & 1 & 2 & 2 & $3(1)^{*}$ & $8(15.4 \%)$ \\
C. glabrata & 1 & 1 & 1 & $2(1)^{*}$ & $5(9.6 \%)$ \\
C. parapsilosis & - & 1 & $3(1)^{\star}$ & 2 & $6(11.5 \%)$ \\
C.guilliermondii & - & - & 1 & - & $1(1.9 \%)$ \\
C. famata & - & - & - & 1 & $1(1.9 \%)$ \\
Total & $8(15.4 \%)$ & $7(13.5 \%)$ & $19(36.5 \%)$ & $18(34.6 \%)$ & $52(100 \%)$ \\
\hline
\end{tabular}

*indicates the number of isolation of a similar species at these sites on the second surveillance culture.

\section{Statistical analyses}

Data were statistically described in terms of mean \pm standard deviation $( \pm S D$ ), median and range, or frequencies (number of cases) and percentages when appropriate. Comparison of numerical variables between the study groups was done using Chi square $\left(\chi^{2}\right)$ test. Exact test was used instead when the expected frequency was less than 5. Correlation between different variables was done using Spearman's Rank correlation equation. $P$ values less than 0.05 were considered statistically significant. All statistical calculations were done using computer programs SPSS (Statistical Package for the Social Science; SPSS Inc., released 2006. SPSS Statistics for Windows, version 15.0. Chicago, IL, USA).

\section{RESULTS}

Twenty one out of the 50 preterms (42\%) admitted during the study period were colonized by Candida spp. Seventeen (17/50; $34 \%)$ were colonized on admission ( $81 \%$ of the colonized preterms), whereas the colonization of the remaining four preterm babies was detected on the second surveillance culture. Six $(28.6 \%)$ preterm babies were colonized at one site only, whereas 15 preterms $(71.4 \%)$ showed multiple site colonization. Eleven $(52.4 \%)$ preterm babies were colonized at two sites, three $(14.3 \%)$ preterm babies were colonized at three sites and only one $(4.8 \%)$ preterm was colonized at four sites. Four preterms out of the 17 initially colonized by Candida spp. (23.5\%) showed complete absence of Candida colonization on the second surveillance culture, only one of whom had received prophylactic fluconazole. Moreover, eight sites initially colonized by Candida species in seven preterms showed loss of colonization at that site, with persistence or appearance of colonization by similar or different Candida species at other sites on the second surveillance culture.

Candida albicans was the commonest among 52 Candida isolates (31/52; 59.6\%) and the groin followed by the rectum showed highest colonization $(36.5 \%$ and $34.6 \%$, respectively) (Table 1 ).

Resistogram typing for isolates belonging to the same species colonizing the same patient revealed that five preterms (patients 12, 13, 17, 33, 37) harboured an identical biotype strain, eight preterms (patients $7,8,10,14$,
$24,42,43,48)$ harboured two different strains and three preterms (patients $32,36,41$ ) harboured three different strains. In seven preterms (patients $8,12,13,17,33,41$, 43) there was persistence of the same isolate at the same site on the second surveillance culture. In four preterms (patients $32,36,41,48$ ), the initial colonizing strain disappeared, with appearance of another distinct strain at the same site (Table 2).

All Candida albicans isolates (100\%) and 19 (90.5\%) non-albicans Candida isolates produced phospholipase enzyme $(P<0.001)$. On the other hand, only two $(6.5 \%)$ Candida albicans versus seven (33.3\%) non-albicans Candida produced proteinase enzyme in vitro $(P=0.022)$. Furthermore, 29 (93.5\%) Candida albicans and 21 (100\%) non- albicans Candida produced biofilm $(P=0.214)$. The degree of phospholipase, proteinase and biofilm production by the Candida isolates is shown in Table 3 . In addition, $26(83.9 \%)$ Candida albicans and 13 (62\%) nonalbicans Candida isolates produced pseudohyphae. The mean value of the pseudohyphae formation percentage was $11.58 \pm 8.563$ for Candida albicans versus $7.24 \pm$ 7.667 for the non-albicans isolates $(P=0.04)$. Among the non-albicans Candida isolates, 7/8 Candida tropicalis, 5/6 Candida parapsilosis and only 1/5 Candida glabrata produced pseudohyphae. Also, 30 (96.8\%) Candida albicans and $14(66.7 \%)$ non-albicans Candida isolates produced coagulase enzyme $(P=0.005)$.

Regarding their susceptibility to antifungal drugs, there was no statistically significant difference between the Candida albicans isolates and the non-albicans Candida towards fluconazole when grown as planktonic cells $(P=$ 1.00 ), whereas $C$. albicans became significantly more resistant to fluconazole compared to the non-albicans Candida isolates when grown into biofilm $(P<0.001)$. Similarly, all Candida species were susceptible to ampB in planktonic form, but when grown into biofilm, the nonalbicans Candida isolates were significantly more susceptible than $C$. albicans $(P=0.007)$ (Table 4$)$.

Both coagulase and phospholipase production strongly correlated with the resistance of sessile Candida isolates to ampB. On the other hand, both proteinase and phospholipase correlated with the resistance of in vitro Candida biofilms to FCZ (Table 5). 
Table 2. Resistograms of Candida isolates belonging to the same species within preterms.

\begin{tabular}{|c|c|c|c|c|c|}
\hline \multirow{2}{*}{ Patient no } & \multirow{2}{*}{ Specie } & \multirow{2}{*}{ Number of isolates } & \multicolumn{2}{|c|}{ Site(s) of isolation } & \multirow{2}{*}{ Resistogram of the isolated strain } \\
\hline & & & Initial & day 7 & \\
\hline \multirow{2}{*}{7} & \multirow{2}{*}{ C. albicans } & \multirow{2}{*}{2} & --- & $\mathrm{O}$ & $A B--F$ \\
\hline & & & --- & $G$ & $-B--F$ \\
\hline \multirow{2}{*}{8} & \multirow{2}{*}{ C. albicans } & \multirow{2}{*}{3} & $\mathrm{R}$ & $\mathrm{R}$ & $-B--F$ \\
\hline & & & --- & $\mathrm{G}$ & $A B---F$ \\
\hline \multirow{2}{*}{10} & \multirow{2}{*}{ C. tropicalis } & \multirow{2}{*}{2} & $\mathrm{R}$ & --- & $A B--E-$ \\
\hline & & & --- & G & $A B--E F$ \\
\hline 12 & C. albicans & 2 & 0 & 0 & $-B--F$ \\
\hline 13 & C. tropicalis & 4 & UGR & $\mathrm{R}$ & A B - - E - \\
\hline \multirow{2}{*}{14} & \multirow{2}{*}{ C. tropicalis } & \multirow{2}{*}{2} & 0 & --- & A B - - E - \\
\hline & & & $U$ & --- & $-B--E-$ \\
\hline 17 & C. albicans & 2 & $\mathrm{G}$ & G & $-B--F$ \\
\hline \multirow{3}{*}{24} & \multirow{3}{*}{ C. albicans } & \multirow{3}{*}{2} & --- & G & $-B--F$ \\
\hline & & & --- & $\mathrm{R}$ & $A B--E F$ \\
\hline & & & G & --- & $-B--E F$ \\
\hline \multirow[t]{2}{*}{32} & \multirow[t]{2}{*}{ C. albicans } & \multirow[t]{2}{*}{3} & --- & G & $A B--E F$ \\
\hline & & & --- & $u$ & $-B--F$ \\
\hline \multirow[t]{2}{*}{33} & \multirow[t]{2}{*}{ C. albicans } & \multirow[t]{2}{*}{4} & GR & UG & $-B--F$ \\
\hline & & & UR & --- & $-B--F$ \\
\hline \multirow[t]{2}{*}{36} & \multirow[t]{2}{*}{ C. glabrata } & \multirow[t]{2}{*}{4} & --- & $\mathrm{R}$ & $-B-D E F$ \\
\hline & & & --- & 0 & $A B-D E F$ \\
\hline \multirow[t]{2}{*}{37} & C. albicans & 2 & UR & --- & $A B--E F$ \\
\hline & & & $G$ & $\mathrm{G}$ & $-B--F$ \\
\hline 41 & C. albicans & 4 & $\mathrm{R}$ & ---- & $A B-D E F$ \\
\hline & & & --- & $\mathrm{R}$ & $A B--E F$ \\
\hline 42 & C narancilncic & 2 & G & --- & $-B-E F$ \\
\hline & c. parapsilosis & 2 & $\mathrm{R}$ & ---- & $-B--E-$ \\
\hline 13 & C narancilncis & 3 & G & G & $-B--F$ \\
\hline 40 & 4. parapsilusis & 3 & $\mathrm{R}$ & ---- & $-B--E F$ \\
\hline 48 & C albicans & 3 & $\mathrm{R}$ & ---- & $A B--E F$ \\
\hline & & 3 & --- & GR & $-B--F$ \\
\hline
\end{tabular}

$\mathrm{O}=$ Oral isolate $\mathrm{G}=$ groin isolate $\mathrm{R}=$ rectal isolate $\mathrm{U}=$ umbilical isolate

Table 3. The degree of phospholipase, proteinase and biofilm production by the Candida isolates.

\begin{tabular}{|c|c|c|c|c|c|c|c|c|c|c|}
\hline \multirow{2}{*}{ Specie } & \multicolumn{4}{|c|}{ Phospholipase } & \multicolumn{2}{|c|}{ Proteinase } & \multicolumn{4}{|c|}{ Biofilm } \\
\hline & $(-)$ & $(++)$ & $(+++)$ & $(++++)$ & $(-)$ & $(++)$ & $(-)$ & $(+)$ & $(++)$ & $(+++)$ \\
\hline C. albicans $(\mathrm{n}=31)$ & 0 & 0 & $9(29 \%)$ & $22(76 \%)$ & $29(93.5 \%)$ & $2(6.5 \%)$ & $2(6.5 \%)$ & $2(6.5 \%)$ & $23(74 \%)$ & $4(13 \%)$ \\
\hline Non-albicans Candida $(\mathrm{n}=21)$ & $2(9.5 \%)$ & $5(24 \%)$ & $12(57 \%)$ & $2(9.5 \%)$ & $14(66.7 \%)$ & $7(33 \%)$ & 0 & $4(19 \%)$ & $12(57 \%)$ & $5(24 \%)$ \\
\hline Total $(n=52)$ & $2(3.8 \%)$ & $5(9.6 \%)$ & $21(40 \%)$ & $24(46 \%)$ & $43(82.6 \%)$ & $9(17 \%)$ & $2(3.8 \%)$ & $6(11.5 \%)$ & $35(67 \%)$ & $9(17 \%)$ \\
\hline
\end{tabular}

$(-)=$ no production; $(+)=$ mild production; $(++)=$ moderate production; $(+++)=$ strong production; $(++++)=$ very strong production 
Table 4. Susceptibility of the 52 colonizing Candida isolates to fluconazole and amphotericin B.

\begin{tabular}{|c|c|c|c|c|c|c|c|c|}
\hline \multirow{2}{*}{$\begin{array}{l}\text { Type of } \\
\text { growth }\end{array}$} & \multirow{2}{*}{ Specie } & \multicolumn{4}{|c|}{ Fluconazole } & \multicolumn{3}{|c|}{ Amphotericin B } \\
\hline & & S N (\%) & S-DD N (\%) & R N (\%) & $P$ value & S N (\%) & R N (\%) & $P$ value \\
\hline \multirow[b]{2}{*}{ Planktonic } & C. albicans $(\mathrm{n}=31)$ & $30(96.8 \%)$ & $1(3.2 \%)$ & 0 & \multirow[b]{2}{*}{1.00} & $31(100 \%)$ & 0 & \multirow[b]{2}{*}{$N D^{(c)}$} \\
\hline & $\begin{array}{l}\text { Non-albicans Candida } \\
\text { spp. }(\mathrm{n}=21)\end{array}$ & $20(95.2 \%)$ & $1(4.8 \%)$ & 0 & & $21(100 \%)$ & 0 & \\
\hline \multirow[b]{2}{*}{ Sessile } & C. albicans $(\mathrm{n}=29)^{(a)}$ & $5(17.2 \%)$ & $5(17.2 \%)$ & $19(65.5 \%)$ & \multirow[b]{2}{*}{$<0.001^{(b)}$} & $1(3.4 \%)$ & $28(96.6 \%)$ & \multirow[b]{2}{*}{$0.007^{(b,}$} \\
\hline & $\begin{array}{l}\text { Non-albicans Candida } \\
\text { spp. }(\mathrm{n}=21)\end{array}$ & $15(71.4 \%)$ & $5(23.8 \%)^{(d)}$ & $1(4.8 \%)$ & & $7(33.3 \%)$ & $14(66.7 \%)$ & \\
\hline
\end{tabular}

$S=$ sensitive, $S-D D=$ sensitive dose dependant, $R=$ resistant. ${ }^{\left({ }^{a}\right)}$ Two $C$. albicans isolates were not tested being non-biofilm forming. ${ }^{(b)} P<0.05$ significant. ${ }^{(c)}$ No statistics could be computed. ${ }^{(d)} 25 \%$ of the planktonically susceptible non-albicans Candida became S-DD when grown as a biofilm $(P<0.001)$.

Table 5. Correlation between different virulence factors and resistance of sessile Candida isolates to amphotericin $B$ and fluconazole.

\begin{tabular}{lcc}
\hline Virulence factor & Amphotericin B & Fluconazole \\
\hline Pseudohyphae & 0.084 & 0.274 \\
Coagulase & $0.702^{\star *}$ & 0.183 \\
Phospholipase & $0.603^{\star *}$ & $0.454^{\star *}$ \\
Proteinase & 0.042 & $-0.305^{\star}$ \\
\hline
\end{tabular}

${ }^{*} P<0.05,{ }^{* *} P \leq 0.001$

The MIC data for the isolated Candida spp. are summarized in Table 6. When grown as planktonic cells, FCZ and ampB exhibited good activity against all Candida spp.; with their MIC90 within the susceptible category. The MIC50 and MIC90 values for CHX against all Candida spp. grown as planktonic cells were comparable to FCZ. On the other hand, when grown as biofilm, the MIC90 values for FCZ and ampB increased against all Candida spp and lay within the resistant category. However, the MIC50 for FCZ against non-albicans Candida remained susceptible despite their growth as biofilm, such a value was similar to that of $\mathrm{CHX}$.

\section{DISCUSSION}

The overall Candida colonization among the preterm neonates during the study period was $42 \%$, nearly the same rate as previously reported by Manzoni et al. (2007), but higher than that reported by Ali et al. (2012). Candida albicans was the commonest colonizing species $(59.6 \%)$, which is in agreement with other studies (Oksuz et al., 2007; Ali et al., 2012).

Fifteen preterms $(71.4 \%)$ showed multiple site colonization, which is comparable to the results reported by Huang et al. (2004). Resistotyping is a phenotypic method which allows differentiation of isolates from clinical samples, and has been used in clinical epidemiological studies (Nakamura et al., 1998; Prasobh et al., 2009), with results complementary to genotypic methods (Leung et al., 2000). In the present study, similar species were isolated from the same or different sites of 16 patients in the initial surveillance specimens and/or after seven days. Seven patients harboured identical strains colonizing different sites or the same site after seven days. Eleven patients harboured different strains, whether in addition to identical strains or not. Infants admitted to NICUs may harbour single or multiple Candida strains at multiple sites for prolonged periods.

However, they may occasionally acquire a new Candida strain of either the same or different species from environmental surfaces and the hands of healthcare workers (Huang et al., 2004).

Prophylactic use of fluconazole is effective in reducing the incidence of fungal colonization and fungal systemic infections in preterm neonates (Manzoni et al., 2007). This was demonstrated in the present study in one initially colonized preterm who received fluconazole prophylactically and showed complete absence of Candida colonization on the second surveillance culture. Nevertheless, three other initially colonized preterms became non-colonized by Candida spp. on the second surveillance culture without receiving any prophylaxis. It might be possible to presume that despite being premature, preterm neonates' immune system may play a role. Phagocytosis of Candida spp. is thought to be a powerful mechanism to control tissue invasion. In absence of specific-antibody mediated opsonization, Candida surface molecules are recognized by membrane-associated carbohydrate-binding lectins expressed by innate immune cells providing important mucosal antifungal mechanism in immunocompromised hosts (Plantinga et al., 2009). Moreover, unconventional immune cells (that is epithelial and endothelial cells) may act as potential anti-Candida effector cells (Maródi and Johnston, 2007).

Several virulence factors enable Candida to cause infection in susceptible hosts (Mohan das and Ballal, 2008). Our study revealed the production of many potential virulence factors by the colonizing Candida isolates. 
Table 6. The in vitro antifungal activity of fluconazole, amphotericin B and chlorhexidine against planktonic and sessile Candida isolates.

\begin{tabular}{|c|c|c|c|c|c|c|}
\hline \multirow{2}{*}{ Drug and Strain } & \multicolumn{3}{|c|}{ Planktonic MIC values in $\mu \mathrm{g} / \mathrm{ml}$} & \multicolumn{3}{|c|}{ Sessile MIC values in $\mu \mathrm{g} / \mathrm{ml}$} \\
\hline & Range & MIC50 & MIC90 & Range & MIC50 & MIC90 \\
\hline \multicolumn{7}{|l|}{ Fluconazole } \\
\hline C. albicans & $<0.12-16$ & $<0.12$ & 8 & $2->64$ & 64 & $>64$ \\
\hline Non-albicans Candida spp. & $<0.12-16$ & $<0.12$ & $<0.12$ & $<0.12-64$ & $<0.12$ & 32 \\
\hline \multicolumn{7}{|l|}{ Amphotericin B } \\
\hline C. albicans & $<0.03-0.5$ & $<0.03$ & 0.5 & $<0.03->16$ & 8 & 8 \\
\hline Non-albicans Candida spp. & $<0.03-0.25$ & $<0.03$ & 0.06 & $<0.03-16$ & 8 & 8 \\
\hline \multicolumn{7}{|l|}{ Chlorhexidine } \\
\hline C. albicans & $<0.12-16$ & 4 & 8 & $<0.12-32$ & 8 & 16 \\
\hline Non-albicans Candida spp. & $<0.12-8$ & $<0.12$ & 8 & $<0.12-32$ & $<0.12$ & 16 \\
\hline
\end{tabular}

To begin with, 50/52 (96\%) Candida isolates produced phospholipase, which was significantly higher in Candida albicans. Similar findings have been reported by other investigators (Oksuz et al., 2007; Issa et al., 2011). Moreover, high phospholipase production, which might reflect a more virulent nature (Mohan das and Ballal, 2008), was demonstrated in 22/31 (71\%) Candida albicans. This is comparable to a study performed on blood culture isolates (Gokce et al., 2007), but is much higher than another study performed on colonizing isolates (Oksuz et al., 2007). On the other hand, our study revealed significantly more non-albicans Candida producing proteinase than Candida albicans producing proteinase (33.3\% versus $6.5 \%, P=0.022$ ). This agrees with the work of Mohan das and Ballal (2008) on blood culture isolates, but is contradictory to the work of others studying either colonizing or clinical isolates (Oksuz et al., 2007; Gokce et al., 2007; Issa et al., 2011); where the percentage of $C$. albicans producing proteinase was significantly higher than non-albicans Candida.

Biofilm formation represents a protected mode of growth that allows cells to survive and disperse to colonize new niches (Hall-Stoodley et al., 2004). It has been shown that non-albicans Candida are more frequent biofilm producers than C. albicans (Gokce et al., 2007). The present study detected biofilm production among 93.5\% Candida albicans and all the non-albicans Candida, with no significant difference between them $(P=0.214)$. The present study also showed that $83.9 \% C$. albicans and $62 \%$ non-albicans Candida produce pseudohyphae, with a mean pseudohyphae percentage significantly higher in C. albicans $(P=0.04)$. Pseudohyphae production has been considered necessary for the virulence of $C$. albicans (Du et al., 2012), and has been demonstrated in certain non-albicans Candida spp. (Negri et al., 2010). Coagulase production may also be related to the pathogenicity of Candida spp. (Yigit et al., 2008). Our study showed the ability of $96.8 \%$ C. albicans and $66.7 \%$ nonalbicans Candida isolates to produce coagulase enzyme
$(P=0.005)$. Similar results have been reported by others (Rodrigues et al., 2003; Yigit et al., 2008).

Management guidelines for neonatal candidiasis recommend treatment with amphotericin B deoxycholate and suggest treatment with fluconazole or amphotericin B lipid products as alternatives (Pappas et al., 2009). Grown as planktonic cells, none of the isolates exhibited resistance towards both antifungals. Our results are comparable with the results of Issa et al. (2011).

Most of our isolates exhibited an ability to form biofilm, therefore their biofilm susceptibility was tested. In total, $40 \%$ and $84 \%$ Candida isolates grown into biofilm became resistant to fluconazole and amphotericin $\mathrm{B}$, respectively. There was also a significant change regarding non-albicans Candida and fluconazole; where $25 \%$ fluconazole susceptible planktonic non-albicans Candida became susceptible but dose dependant when grown into biofilm. Similar results regarding ampB have been reported (Ramage et al., 2001; Jain et al., 2007); where ampB has demonstrated some activity against Candida biofilms. However, these two studies have shown that Candida biofilms display total resistance to FCZ. Basic physical barriers such as extracellular matrix and cell density have been shown to produce recalcitrance to antifungal agents (Ramage et al., 2012). It is to be noted that only $13 \%$ C. albicans and $24 \%$ non-albicans Candida were strong biofilm producers in our study, which might explain the difference regarding FCZ. Moreover, C. albicans sessile cells were more resistant to both antifungals compared to non-albicans Candida; $65.5 \%$ versus $4.8 \%$ and $96.6 \%$ versus $66.7 \%$ for FCZ and ampB, respectively. Again, this might be explained by difference in biofilm; Kuhn et al. (2002a) demonstrated by dry weight measurement and microscopic analyses that $C$. albicans consistently produced more biofilm than non-albicans Candida; whose biofilms appeared less thick, formed of only basal blastospore layers with minimal extracellular matrix and hyphae.

Studies on planktonically grown Candida albicans have shown correlation between high activity of each of phos- 
phospholipase as well as proteinase and resistance to antifungal drugs (Kumar and Shukla, 2010; Ying and Chunyang, 2012). Nevertheless, the present study showed absence of resistance of planktonically grown Candida isolates towards both antifungals. However, there was a positive correlation between the degree of phospholipase production and the antifungal drug resistance of the isolates grown as biofilm. Similarly, coagulase production correlated with biofilm resistance to amphotericin B. Interestingly, a negative correlation was noted between proteinase production and biofilm resistance to fluconazole; where none of the isolates resistant to fluconazole when gown as biofilm were_proteinase producers. Biofilm production and proteinase secretion have been found to be negatively correlated, possibly due to the role of proteinase in the degradation of extracellular matrix (Tavanti et al., 2010), a major contributor to drug resistance in Candida biofilms (Ramage et al., 2012). Based on our results, it might be possible to suggest that biofilm per se does not necessarily lead to antifungal resistance, but it is the coordination of certain virulence factors in the presence of biofilm production that results in resistance.

Chlorhexidine is a cationic biguanide, with low mammalian toxicity and an ability to bind to skin and mucous membranes (Hope and Wilson, 2004). It has a broad spectrum of activity against a variety of micro-organisms. However, biofilm susceptibility to chlorhexidine has been shown to be significantly reduced compared to its action against planktonic cells (Suci and Tyler, 2002). In the present study, chlorhexidine was tested against the isolated Candida spp. Taken as a whole, there was similar potency of chlorhexidine and fluconazole against all the planktonically grown Candida isolates, with an MIC range of $<0.12$ to $16 \mu \mathrm{g} / \mathrm{ml}$, whereas amphotericin $B$ had much less values. However, grown as sessile cells, the MIC range of chlorhexidine did not show much change, becoming comparable to the results of amphotericin $B$ which was much less than the MIC ranges for fluconazole. There was no change in the chlorhexidine MIC50 value for non-albicans Candida whether planktonic or sessile, whereas the chlorhexidine MIC50 and MIC90 values of $C$. albicans doubled when grown as sessile form. Similar MIC values for chlorhexidine against planktonically grown Candida isolates of different species have been reported (Traboulsi et al., 2008). On the other hand, higher MIC ranges of 16 to $32 \mu \mathrm{g} / \mathrm{ml}$ have been reported by Tobudic et al. (2008). However, the same study reported inability to retrieve any viable cell of Candida spp from biofilms when treated with $0.25 \%$ chlorhexidine. Another study revealed an eight times increase in the MIC range for chlorhexidine towards sessile forms of Candida spp. (Kuhn et al., 2002b). Nevertheless, the authors showed that local low levels of chlorhexidine may be sufficient to inhibit or disrupt biofilm formation which might be of value for prophylaxis.

The present study is limited by only examining Candida isolates colonizing preterms, without addressing risk factors associated with colonization or development of ICls. However, none of the included preterms died during the period of surveillance cultures.

In conclusion, Candida spp. colonizing preterms possessed various potential virulence factors and consequently might be able to cause infection under favourable conditions. On the whole, fluconazole demonstrated better activity than amphotericin B against the colonizing Candida isolates. Phospholipase and coagulase correlated with the development of biofilm drug resistance. Chlorhexidine demonstrated good in vitro activity towards planktonic and sessile Candida isolates, which might be promising in the eradication of Candida colonization and requires future assessment through clinical trials.

\section{REFERENCES}

Ali GY, Algohary EH, Rashed KA, Almoghanum M, Khalifa AA (2012). Prevalence of Candida colonization in preterm newborns and VLBW in neonatal intensive care unit: role of maternal colonization as a risk factor in transmission of disease. J. Matern. Fetal Neonatal. Med. 25:789-795.

Clinical and Laboratory Standards Institute, CLSI/National Committee for Clinical Laboratory Standards (2008). Reference method for broth dilution susceptibility testing for yeasts. $3^{\text {rd }}$ ed. Approved Standard. Document M27-A3. Wayne, Pa: Clinical and Laboratory Standards Institute.

Du H, Guan G, Xie J, Sun Y, Tong Y, Zhang L, Huang G (2012). Roles of Candida albicans Gat2, a GATA-Type Zinc Finger Transcription Factor, in biofilm formation, filamentous growth and virulence. PLoS One 7: e29707

Fathilah AR, Himratul-Aznita WH, Fatheen ARN, Suriani KR (2012). The antifungal properties of chlorhexidine digluconate and cetylpyrinidinium chloride on oral Candida. J. Dent. 40: 609-615.

Gokce G, Cerkcioglu N, Yagci A (2007). Acid proteinase, phospholipase, and biofilm production of Candida species isolated from blood cultures. Mycopathologia 164: $265-269$.

Hall-Stoodley L, Costerton JW, Stoodley P (2004). Bacterial biofilms: from the natural environment to infectious disease. Nat. Rev. Microbiol. 2: 95 - 108.

Hope CK, Wilson M (2004). Analysis of the effects of chlorhexidine on oral biofilm vitality and structure based on viability profiling and an indicator of membrane integrity. Antimicrob. Agents Chemother. 48: 1461-1468.

Howell S, Hazen K (2011). Candida, Cryptococcus and other yeasts of medical importance. In Versalovic et al. (eds) Manual of Clinical Microbiology. 10th edition. ASM Press, Washington DC, USA. pp. $1793-1821$

Huang Y, Su L, Wu T, Lin T (2004). Genotyping analysis of colonizing candidal isolates from very-low-birth weight infants in a neonatal intensive care unit. J. Hosp. Infect. 58:200-203.

Issa SY, Badran EF, Aqel KF, Shehabi AA (2011). Epidemiological characteristics of Candida species colonizing oral and rectal sites of Jordanian Infants. BMC Pediatr. 11:79 - 84.

Jain N, Kohli R, Cook E, Gialanella P, Chang T, Fries BC (2007). Biofilm formation by and antifungal susceptibility of Candida isolates from urine. Appl. Environ. Microbiol. 73:1697-1703.

Kuhn DM, Chandra J, Mukherjee PK, Ghannoum MA (2002a). Comparison of biofilms formed by Candida albicans and Candida parapsilosis on bioprosthetic surfaces. Infect. Immun. 70: 878 - 888.

Kuhn DM, George T, Chandra J, Mukherjee PK, Ghannoum MA (2002b). Antifungal susceptibility of Candida biofilms: Unique efficacy of amphotericin B lipid formulations and echinocandins. Antimicrob. Agents Chemother. 46: $1773-1780$.

Kumar R, Shukla PK (2010). Amphotericin B resistance leads to enhanced proteinase and phospholipase activity and reduced germ 
tube formation in Candida albicans. Fungal Biol. 114: 189 - 197.

Leibovitz E (2012). Strategies for prevention of neonatal candidiasis. Pediatr. Neonatol. 53: $83-89$

Leung WK, Dassanayake RS, Yau JY, Jin LJ, Yam WC, Samaranayake LP (2000). Oral colonization, phenotypic, and genotypic profiles of Candida species in irradiated, dentate, xerostomic nasopharyngeal carcinoma survivors. J. Clin. Microbiol. 38: 2219 - 2226.

Manzoni P, Farina D, Galletto P, Leonessa M, Priolo C, Arisio R, Gomirato $G$ (2007). Type and number of sites colonized by fungi and risk of progression to invasive fungal infection in preterm neonates in neonatal intensive care unit. J. Perinat. Med. 35: 220-226.

Manzoni P, Jacqz-Aigrain E, Rizzollo S, Franco C, Stronati M, Mostert M, Farina D (2011). Antifungal prophylaxis in neonates. Early Hum. Dev. 87: S59-60.

Maródi L, Johnston RB Jr (2007). Invasive Candida species disease in infants and children: occurrence, risk factors, management, and innate host defense mechanisms. Curr. Opin. Pediatr. 19: 693-697.

Mendiratta DK, Rawat V, Thamke D, Chaturvedi P, Chhabra S, Narang $P$ (2006). Candida colonization in preterm babies admitted to neonatal intensive care unit in the rural setting. Indian J. Med. Microbiol. 24: $263-267$.

Mohan das V, Ballal M (2008). Proteinase and phospholipase activity as virulence factors in Candida species isolated from blood. Rev. Iberoam. Micol. 25: $208-210$.

Nakamura K, Ito-Kuwa S, Nakamura Y, Aoki S, Vidotto V, Sinicco A (1998). Resistogram typing of oral Candida albicans isolates from normal subjects in three successive trials. Rev. Iberoam. Micol. 15: 19-21.

Negri M, Martins M, Henriques M, Svidzinski T, Azeredo J, Oliveira R (2010). Examination of potential virulence factors of Candida tropicalis clinical isolates from hospitalized patients. Mycopathologia. 169: $175-182$

Nguyen MH, Clancy CJ, Yu VL, Yu YC, Morris AJ, Snydman DR, Sutton DA, Rinaldi MG (1998). Do in vitro susceptibility data predict the microbiologic response to amphotericin B? Results of a prospective study of patients with Candida fungemia. J. Infect. Dis. 177: 425 430.

Oksuz S, Sahin I, Yildirim M, Gulcan A, Yavuz T, Kaya D, Koc A (2007). Phospholipase and proteinase activities in different Candida species isolated from anatomically distinct sites of healthy adults. Jpn. J. Infect. Dis. 60: 280-283.

Pappas PG, Kauffman CA, Andes D, Benjamin Dk Jr, Calandra T, Edwards JE Jr, Filler SG, Fisher JF, Kullberg BJ, Ostrosky-Zeichner L, Reboli AC, Rex JH, Walsh TJ, Sobel JD, Infectious Diseases Society of America (2009). Clinical practice guidelines for the management of candidiasis: 2009 update by the Infectious Diseases Society of America. Clin. Infect. Dis. 48: 503-535.

Plantinga TS, van der Velden WJ, Ferwerda B, van Spriel AB, Adema G, Feuth T, Donnelly JP, Brown GD, Kullberg BJ, Blijlevens NM, Netea MG (2009). Early stop polymorphism in human dectin-1 is associated with increased Candida colonization in hematopoietic stem cell transplant recipients. Clin. Infect. Dis. 49:724-732.

Prasobh KK, Udhaya V, Lakshmi Sarayu Y (2009). Resistotyping of Candida albicans isolated from sputum samples of patients attending TB clinics in and around Chidambaram. Recent Res. Sci. Technol. $1:$ 180-183.
Ramage G, Rajendran R, Sherry L, Williams C (2012). Fungal Biofilm Resistance. Int. J. Microbiol. doi: 10.1155/2012/528521.

Ramage G, Vande Walle K, Wickes BL, López-Ribot JL (2001). Standardized method for in vitro antifungal susceptibility testing of Candida albicans biofilms. Antimicrob. Agents Chemother. 45: 24752479.

Repp KK., Menor SA, Pettit RK (2007). Microplate alamar blue assay for susceptibility testing of Candida albicans biofilms. Med. Mycol. 45: $603-607$.

Rodrigues AG, Pina-Vaz C, Costa-de-Oliveira S, Tavares C (2003). Expression of plasma coagulase among pathogenic Candida species. J. Clin. Microbiol. 41:5792-5793.

Seneviratne CJ, Jin L, Samaranayake LP (2008). Biofilm lifestyle of Candida: a mini review. Oral Dis. 14: 582-590.

Shin JH, Kee SJ, Shin MG, Kim SH, Lee SK, Suh SP, Ryang DW (2002). Biofilm production by isolates of Candida species recovered from nonneutropenic patients: Comparison of bloodstream isolates with isolates from other sources. J. Clin. Microbiol. 40: 1244- 1249.

Soares AF, de Aquino AL, de Carvallo CP, Nonaka CFW, Almeida D, Pereira Pinto $L$ (2011). Frequency of oral mucositis and microbiological analysis in children with acute lymphoblastic leukemia treated with $0.12 \%$ chlorhexidine gluconate. Braz. Dent. J. 22: $312-316$.

Soma VL, Qin X, Zhou C, Adler A, Berry JE, Zerr DM (2012). The effects of daily chlorhexidine bathing on cutaneous bacterial isolates: A pilot study. Infect. Drug Resist. 5: $75-78$.

Suci PA and Tyler BJ (2002). Action of chlorhexidine digluconate against yeast and filamentous forms in an early-stage Candida albicans biofilm. Antimicrob. Agents Chemother. 46: 3522 - 3531.

Tavanti A, Hensgens LA, Mogavero S, Majoros L, Senesi S, Campa M (2010). Genotypic and phenotypic properties of Candida parapsilosis sensu strictu strains isolated from different geographic regions and body sites. BMC Microbiol. 10: 203 - 214.

Tobudic S, Kratzer C, Graninger W, Georgopoulos A (2008). Effect of disinfectants and caspofungin against planktonic and sessile cells of Candida spp. [abstract]: Proceedings of the $13^{\text {th }}$ Scientific Meeting of the European Society of Chemotherapy and Infectious Diseases, December $3^{\text {rd }}-6^{\text {th }}, 2008$ Vienna, Austria. ESC; Abstract no. P22: pp 39.

Traboulsi RS, Mukherjee PK, Ghannoum MA (2008). In vitro activity of inexpensive topical alternatives against Candida spp. isolated from the oral cavity of HIV-infected patients. Int. J. Antimicrob. Agents. 31: 272-276.

Turkova A, Roilides E, Sharland M (2011). Amphotericin B in neonates: deoxycholate or lipid formulation as first-line therapy - is there a 'right' choice? Curr. Opin. Infect. Dis. 24:163 - 171.

Yigit N, Aktas AE, Ayyildiz A (2008). Detection of coagulase activity in pathogenic Candida species. J. Int. Med. Res. 36: 1378 - 1382.

Ying S, Chunyang L (2012). Correlation between phospholipase of Candida albicans and resistance to fluconazole. Mycoses. 55: 50 55. 